\title{
Total inverse transgastric resection with transoral specimen removal
}

\author{
Sebastian H. Lamm • Daniel C. Steinemann • \\ Georg R. Linke · Dietmar Eucker · Thomas Simon • \\ Andreas Zerz $\cdot$ Reinhard Stoll
}

Received: 5 May 2014/Accepted: 5 November 2014/Published online: 25 December 2014

(C) Springer Science+Business Media New York 2014

\begin{abstract}
Background Laparoscopic local excision is accepted for gastrointestinal stromal tumors (GIST) and benign lesions of the stomach. Yet, tumors at the gastroesophageal junction, on the posterior wall, or in the distal antrum are difficult to approach. Such tumors often must be exposed via gastrotomy or using a rendezvous maneuver. Our method of total intragastric laparoscopic resection using 'pneumogastrum', rigid laparoscope, and conventional laparoscopic instruments is described in an intuitive video.

Methods Two cases of total inverse transgastric resection involved resection of a submucosal GIST, one at the front wall of the cardia and the other on the posterior wall of the antrum. The third case required excision of a large
\end{abstract}

Sebastian H. Lamm and Daniel C. Steinemann shared first and contributed equally to this study.

Electronic supplementary material The online version of this article (doi:10.1007/s00464-014-4037-1) contains supplementary material, which is available to authorized users.

S. H. Lamm $(\bowtie) \cdot$ D. C. Steinemann $(\bowtie) \cdot$ D. Eucker .

A. Zerz $\cdot$ R. Stoll

Department of Surgery, Kantonsspital Baselland,

4101 Bruderholz, Switzerland

e-mail: sebastian.lamm@ksbl.ch

D. C. Steinemann

e-mail: daniel.steinemann@gmx.ch

G. R. Linke

Department of General, Visceral and Transplantation Surgery, University Hospital Heidelberg, Im Neuenheimer Feld 110, 69120 Heidelberg, Germany

\section{T. Simon}

Department of General and Visceral Surgery, Klinik Sinsheim, Alte Weibstadter Strasse 2, 74889 Sinsheim, Germany prepyloric cystic lesion leading to a gastric outlet stenosis. After insertion of three trocars under laparoscopic control, a further trocar was introduced into the stomach and 'pneumogastrum' was established. Two additional 5-mm trocars were intragastrally placed. Intragastric endoscopy with a rigid optic provided an excellent view. The tumor was exposed resected with a linear stapler. The specimen was inserted into an Endo Pouch ${ }^{\mathrm{TM}}$ which was sutured to an orally inserted gastric tube. The Endo Pouch ${ }^{\mathrm{TM}}$ was gently pulled transorally. After removal of the intragastric trocars, the entrance points were laparoscopically closed.

Results From the first and second cases, we retrieved GIST tumors. In the third case, we retrieved a gastritis cystica profunda. Postoperative course was uneventful.

Conclusions Gastric GIST should be resected laparoscopically if negative margins are safely achieved regardless of its size. Tumors at the frontwall and exophytic backwall GIST are addressed by laparoscopic wedge resection. Tumors at the gastrojejunal junction, in the prepyloric region, and fundus as well as submucous GIST of the gastric backwall are best approached by intragastric laparoscopic resection. Transoral specimen retrieval is an interesting option in smaller tumors.

Keywords Abdominal - Cancer - Surgical - Technical . Endoscopy

Laparoscopic wedge resection is largely accepted for treatment of gastrointestinal stromal tumors (GIST) and benign lesions of the stomach. However, tumors located near the gastroesophageal junction, on the posterior wall, or in the distal antrum are difficult to locate and approach laparoscopically, and such tumors often must be exposed via gastrotomy [1]. Alternatively, a laparoscopic-endoscopic 
rendezvous maneuver can be used to visualize tumor borders and perform either laparoscopic wedge resection under endoscopic guidance or intragastric resection under laparoscopic control [2,3]. Reports have also described positioning of an intragastric trocar to enable hybrid laparoscopic and endoscopic resection with gastroscopic assistance [4-7]. Such techniques allow the use of conventional laparoscopic instruments, such as stapling devices, for intragastric tumor resection [8]. Here, we describe our method for total intragastric laparoscopic resection using 'pneumogastrum' with visualization, a rigid laparoscope instead of a flexible gastroscope, and conventional laparoscopic instruments.

\section{Methods}

Between April and November 2013, three patients with intragastric lesions underwent total inverse transgastric resection with transoral specimen removal. Two cases involved resection of a submucosal GIST, one at the front wall of the cardia close to the angle of His, and the other on the posterior wall of the antrum. The third case required excision of a large prepyloric cystic lesion due to gastritis cystica profunda leading to a gastric outlet stenosis. Each patient underwent a preoperative gastroduodenoscopy. Laparoscopy was performed under general anesthesia with administration of single-shot antibiotic prophylaxis with cefazolin. Patient was put in the French position. After pneumoperitoneum establishment, three 5-12-mm trocars were inserted based on the requirements and localization of the tumor. With a stay suture placed in the front wall of the stomach, it was approximated to the abdominal wall. Under laparoscopic control, a trocar sleeve and Veress needle were punctured directly through the abdominal wall into the stomach (Fig. 1). Through the sleeve, a 12-mm Versastep $^{\text {TM }}$ trocar (Covidien plc., Dublin, Ireland) was introduced into the stomach and 'pneumogastrum' of $12 \mathrm{mmHg}$ was established. Using the same technique,

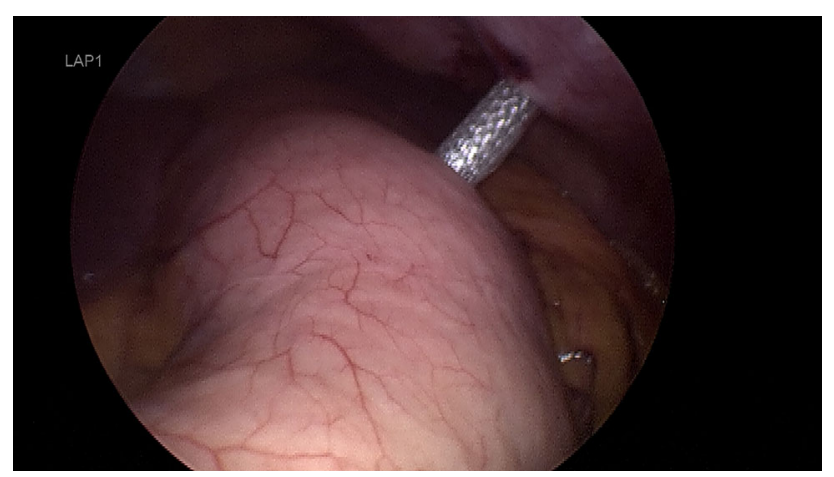

Fig. 1 Laparoscopic control of intragastric trocar introduction under laparoscopic control, two additional 5-mm trocars were intragastrally placed according to the tumor localization (Fig. 2). Intragastric endoscopy with a rigid 5-mm $30^{\circ}$ optic provided an excellent view. The tumor was exposed using a 5-mm endo grasper (Fig. 3) and resected stepwise with up to three $45-\mathrm{mm}$ reloads $(3-4 \mathrm{~mm}$ ) of a linear endo stapler (Fig. 4). The stapler line was controlled for bleeding and possible bleedings were overstitched using Vicryl 3-0 thread with extracorporeal knotting. The specimen was inserted into a $3 \times 6$ inch Endo Pouch ${ }^{\mathrm{TM}}$

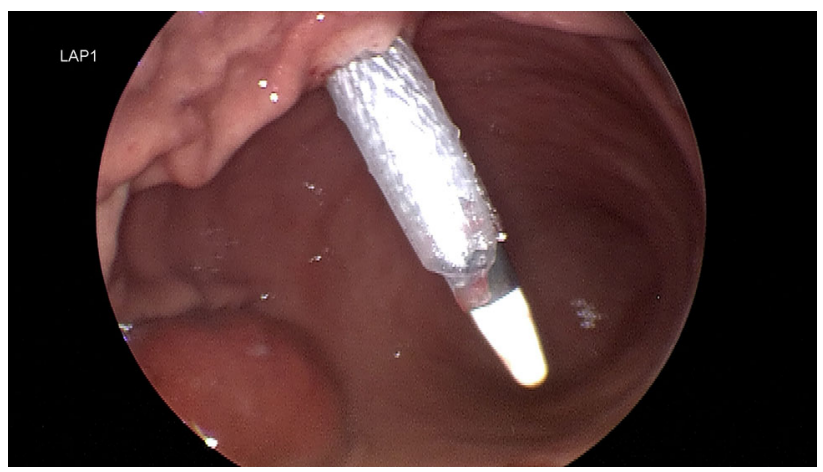

Fig. 2 Trocar introduction into the stomach, controlled from inside the stomach

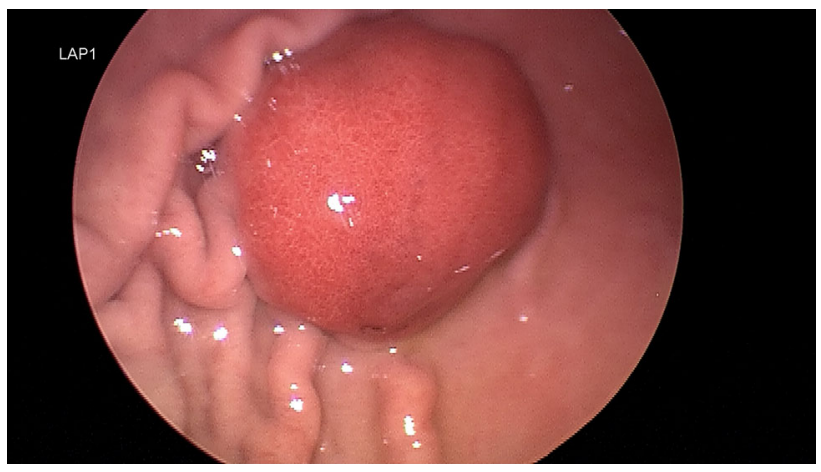

Fig. 3 GIST tumor at the posterior wall of the antrum (second case)

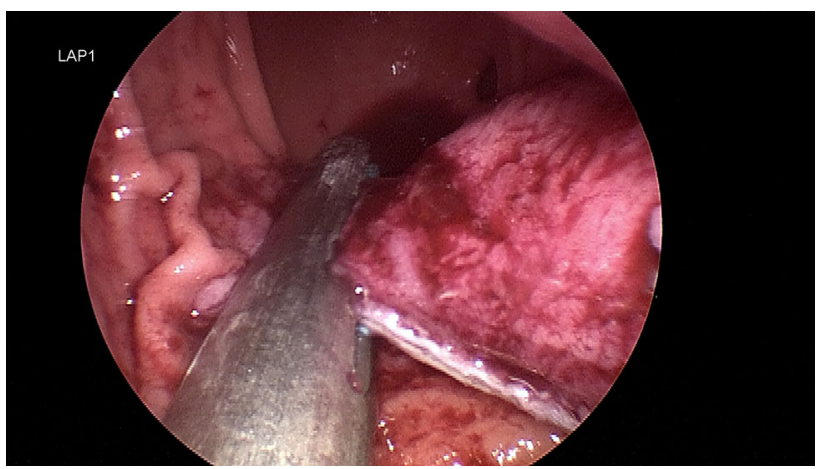

Fig. 4 Resection of GIST tumor by intragastric stapling 
(Unimax Medical Systems Inc., Taipei, Taiwan), a gastric tube was transorally inserted, and the Endo Pouch ${ }^{\mathrm{TM}}$ suture was threaded through the gastric tube side openings and knotted to fix the Endo Pouch ${ }^{\mathrm{TM}}$ to the gastric tube. Then the Endo Pouch ${ }^{\mathrm{TM}}$ was gently pulled transorally, the intragastric trocars were removed, and the entrance points at the anterior stomach wall were laparoscopically closed with Vicryl 2-0 running sutures. The pneumoperitoneum was deflated and the remaining trocars were removed.

\section{Results}

From the first and second cases, we retrieved GIST tumors (pT2) with diameters of 26 and $21 \mathrm{~mm}$, respectively. In the third case, we retrieved a $25 \times 15 \times 13 \mathrm{~mm}$ prepyloric specimen containing a gastritis cystica profunda. All three patients experienced uneventful postoperative courses.

\section{Conclusions}

Local excision of benign lesions and GIST in the stomach is a well-accepted practice. The National Cancer Care Network Guidelines (NCCNG), the guidelines of the Japan Society of Clinical Oncology (JSCO), and the consensus conference 2004 of the European Society for Medical Oncology (ESMO) all agree that GIST should be resected with an intact pseudocapsule and negative microscopic margins, with all possible measures taken to avoid tumor rupture. There are reportedly no benefits to formal lymph node resection or wider resection [9-11]. Compared to the laparoscopic resection of smaller tumors, laparoscopy for GIST of over $5 \mathrm{~cm}$ in diameter may be considered more technically challenging [12]. However, if tumor rupture can be safely prevented and complete resection is achievable, there is no formal contraindication for laparoscopic resection in such cases [9]. Recent studies have reported no differences in oncological safety and long-term oncological outcome between laparoscopic and open resections of GIST, including a systematic review comparing 381 laparoscopic versus 384 open procedures [13, 14].

The most commonly performed laparoscopic technique for gastric GIST is a wedge resection. The systematic review by Koh et al. [13] reported that this procedure carried a conversion rate as low as $4.7 \%$. The wedge resection is technically uncomplicated for an experienced laparoscopic surgeon and has been successfully performed for tumors measuring up to $19 \mathrm{~cm}$ in diameter. The largest tumors resected with this procedure have been lying subserosal and have had a pedunculated shape [14-17]. Localization of submucosal GIST may be facilitated using additional intraoperative gastroscopic guidance. With endoscopic assistance, the half-circumference of the tumor is laparoscopically dissected neuromuscularly. The mobilized GIST can then be exteriorized and resected using a laparoscopic stapler [18-20]. GIST of the gastric back wall with an exophytic subserous growth pattern that can be clearly visualized laparoscopically may be resected without endoscopic control. Giant GIST of the greater curvature are also amenable to gastric sleeve resection [21].

Lesions near the gastroesophageal junction, the prepyloric area, and the fundus are difficult to reach by laparoscopic wedge resection. Furthermore, it can be challenging to avoid a stenosis. GIST in those locations, as well as submucous tumors of the gastric back wall, can best be approached via anterior gastrotomy, followed by tissuesaving-stapled wedge resection from the inside [22, 23]. Reports have described the use of the DaVinci surgical system (Intuitive Surgical Inc., Sunnyvale CA, USA) for wedge resection in the posterior wall, followed by closure with easily applicable sutures [24].

In the present case series, we describe a technique in which gastrotomy is avoided by placement of intragastric trocars. This method enables optimal visualization of the critical anatomic structures during local resection of GIST with difficult localization [25-27]. Additionally, the potential complications of gastrotomy closure are avoided and the risk of intraperitoneal contamination is minimized. Intragastric resection without gastrotomy makes the operation even less invasive.

A review of 51 cases out of seven case series of intragastric laparoscopic resection demonstrated the feasibility of this technique. Of those cases, 28 were localized in the gastroesophageal junction, 4 in the fundus, and 19 in the posterior wall. Tumor sizes ranged between 1.3 and $7.5 \mathrm{~cm}$. Of the 51 resections, $3(5.9 \%)$ had to be converted to open procedures [25]. More recently, intragastric resection using a single-incision trocar has been reported for resection of GIST of $6.5 \mathrm{~cm}$ in diameter and localized near the gastroesophageal junction [28]. The technique used in this case series employed optimal rigid standard laparoscopic instruments, including endo-staplers and laparoscopic suturing devices.

Most authors advocate that the intragastric procedure should be assisted by gastroscopy and transoral specimen retrieval [25]. For larger tumors, an alternative route of specimen retrieval is via the transgastric trocar through the abdominal wall [28]. For the resection of large GIST, one author has described morcellation of the tumor in the removal bag to allow transoral retrieval [29]. However, in our opinion, morcellation prevents adequate pathological examination of the resection margin and is therefore not recommended and not in accordance with the aforementioned guidelines. In our experience, the use of a rigid laparoscopic-endoscope instead of a gastroscope optimizes 
lesion visualization and exposure. Furthermore, this technique eliminates the need for gastroscopy and rendezvous maneuver. In the presently reported cases, transoral specimen removal was possible without gastroscopy. With transoral extraction, the procedure is fully laparoscopic, leaving only small trocar scars. This procedure may also reduce postoperative pain and enable faster recovery.

In summary, the available guidelines state that gastric GIST of any size should be resected laparoscopically if negative margins can be safely achieved and tumor rupture can be avoided. Tumors at the gastric front wall and exophytic GIST of the gastric back wall are typically removed by laparoscopic wedge resection. Endoluminal GIST are resected using a half-exteriorized technique with assistance of gastroscopic visualization. For GIST in difficult locations, such as the gastrojejunal junction, prepyloric region, and gastric fundus, and for submucous GIST of the gastric back wall, the best approach is by intragastric laparoscopic resection. In such cases, transoral specimen retrieval is a good option if the tumor size allows easy passage through the lower esophageal sphincter.

Disclosures Drs. Sebastian H. Lamm, Daniel C. Steinemann, Georg R. Linke, Dietmar Eucker, Thomas Simon, Andreas Zerz, and Reinhard Stoll have no conflicts of interest or financial ties to disclose.

\section{References}

1. Warsi AA, Peyser PM (2010) Laparoscopic resection of gastric GIST and benign gastric tumours: evolution of a new technique. Surg Endosc 24(1):72-78

2. Maker AV (2013) A technique for laparoendoscopic resection of posterior fundic gastric GISTs without need for a gastrotomy. Ann Surg Oncol 20(13):4238

3. Schubert D et al (2005) Laparoscopic-endoscopic rendezvous resection of upper gastrointestinal tumors. Dig Dis 23(2):106-112

4. Shim JH et al (2011) Intragastric approach for submucosal tumors located near the Z-line: a hybrid laparoscopic and endoscopic technique. J Surg Oncol 104(3):312-315

5. Sahm M, Pross M, Lippert H (2011) Intraluminal resection of gastric tumors using intragastric trocar technique. Surg Laparosc Endosc Percutaneous Tech 21(4):e169-e172

6. Walsh RM et al (2003) Combined endoscopic/laparoscopic intragastric resection of gastric stromal tumors. J Gastrointest Surg 7(3):386-392

7. Uchikoshi $\mathrm{F}$ et al (2004) Laparoscopic intragastric resection of gastric stromal tumor located at the esophago-cardiac junction. Surg Laparosc Endosc Percutaneous Tech 14(1):1-4

8. Tagaya $\mathrm{N}$ et al (2002) Laparoscopic intragastric stapled resection of gastric submucosal tumors located near the esophagogastric junction. Surg Endosc 16(1):177-179

9. Demetri GD et al (2010) NCCN Task Force report: update on the management of patients with gastrointestinal stromal tumors. J Natl Compr Cancer Netw 8(Suppl 2):S1-S41 (quiz S42-4)
10. Blay JY et al (2005) Consensus meeting for the management of gastrointestinal stromal tumors. Report of the GIST Consensus Conference of 20-21 March 2004, under the auspices of ESMO. Ann Oncol 16(4):566-578

11. Nishida $T$ et al (2008) Clinical practice guidelines for gastrointestinal stromal tumor (GIST) in Japan: english version. Int J Clin Oncol 13(5):416-430

12. Nishimura J et al (2007) Surgical strategy for gastric gastrointestinal stromal tumors: laparoscopic vs. open resection. Surg Endosc 21(6):875-878

13. Koh YX et al (2013) A systematic review and meta-analysis comparing laparoscopic versus open gastric resections for gastrointestinal stromal tumors of the stomach. Ann Surg Oncol 20(11):3549-3560

14. Valle $M$ et al (2014) Gastrointestinal stromal tumors of the stomach: the role of laparoscopic resection. Single-centre experience of 38 cases. Surg Endosc 28(3):1040-1047

15. Kim DJ, Lee JH, Kim W (2014) Laparoscopic resection for 125 gastroduodenal submucosal tumors. Ann Surg Treat Res 86(4):199-205

16. Anania G et al (2013) Totally laparoscopic resection of a very large gastric GIST. G Chir 34(7-8):227-230

17. De Vogelaere K et al (2012) Laparoscopic resection of gastric gastrointestinal stromal tumors (GIST) is safe and effective, irrespective of tumor size. Surg Endosc 26(8):2339-2345

18. Wilhelm D et al (2008) Simultaneous use of laparoscopy and endoscopy for minimally invasive resection of gastric subepithelial masses-analysis of 93 interventions. World J Surg 32(6): 1021-1028

19. Kakeji Y et al (2012) Laparoscopic resection for gastrointestinal stromal tumors in the stomach. Surg Today 42(6):554-558

20. Hiki $\mathrm{N}$ et al (2008) Laparoscopic and endoscopic cooperative surgery for gastrointestinal stromal tumor dissection. Surg Endosc 22(7):1729-1735

21. Sokolich J et al (2009) Expanding the indications for laparoscopic gastric resection for gastrointestinal stromal tumors. JSLS 13(2):165-169

22. Pucci MJ et al (2012) Laparoscopic approaches to gastric gastrointestinal stromal tumors: an institutional review of 57 cases. Surg Endosc 26(12):3509-3514

23. Sakamoto Y et al (2012) Safe laparoscopic resection of a gastric gastrointestinal stromal tumor close to the esophagogastric junction. Surg Today 42(7):708-711

24. Moriyama H et al (2012) Robot-assisted laparoscopic resection for gastric gastrointestinal stromal tumor. Surg Laparosc Endosc Percutaneous Tech 22(3):e155-e156

25. Li VK et al (2008) Laparoscopic intragastric approach for stromal tumours located at the posterior gastric wall. Asian J Surg 31(1):6-10

26. Vecchio R et al (2013) Laparoscopic-endoscopic rendez-vous resection of iuxta-cardial gastric GIST. G Chir 34(5-6):145-148

27. Conrad $\mathrm{C}$ et al (2014) Techniques of intragastric laparoscopic surgery. Surg Endosc. doi:10.1007/s00464-014-3654-z

28. De Vogelaere K et al (2013) Intragastric SILS for GIST, a new challenge in oncologic surgery: first experiences. Anticancer Res 33(8):3359-3363

29. Huscher CG et al (2013) Transoral extraction of a laparoscopically resected large gastric GIST. J Laparoendosc Adv Surg Tech A 23(8):707-709 